\title{
Multi-strain Probiotic Increases the Gut Microbiota Diversity in Obese Pregnant Women: Results from a Randomized, Double-blind Placebo-controlled Study
}

\section{Sofie Ingdam Halkjær}

Hvidovre Hospital https://orcid.org/0000-0001-7518-4252

Victoria Elisabeth de Knegt

Hvidovre Hospital

Bobby Lo

Hvidovre Hospital

Lisbeth Nilas

Hvidovre Hospital

Dina Cortes

Hvidovre Hospital

Anders Elm Pedersen

Kobenhavns Universitet Sundhedsvidenskabelige Fakultet

Hengameh Chloé Mirsepasi-Lauridsen

Statens Serum Institut

Lee O'Brien Andersen

Statens Serum Institut

Henrik Vedel Nielsen

Statens Serum Institut

Christen Rune Stensvold

Statens Serum Institut

Thor Bech Johannesen

Statens Serum Institut

Thomas Kallemose

Hvidovre Hospital

Karen Angeliki Krogfelt

Roskilde Universitet

Andreas Munk Petersen ( $\nabla$ andreas.munk.petersen@regionh.dk)

Hvidovre Hospital https://orcid.org/0000-0003-0531-0553 
Keywords: gestational diabetes mellitus, gestational weight gain, microbiota, obesity, pregnancy, probiotics

Posted Date: December 3rd, 2019

DOI: https://doi.org/10.21203/rs.2.18046/v1

License: (c) (1) This work is licensed under a Creative Commons Attribution 4.0 International License. Read Full License 


\section{Abstract}

Background: Maternal obesity is associated with adverse pregnancy outcomes. Probiotic supplementation during pregnancy may have positive effects on blood glucose, gestational weight gain (GWG), and the risk of gestational diabetes mellitus (GDM). The primary aim was to determine the feasibility of probiotic intervention in obese pregnant women from the early second trimester until delivery. The secondary aim was to investigate the effect of daily probiotic supplementation on GWG, maternal glucose homeostasis, infant birthweight, and maternal gut microbiota. We carried out a randomized double-blinded placebo-controlled study in 50 obese pregnant women. Participants were randomly allocated to two treatment groups, multi-strain probiotic [Vivomixx $\circledast$ ] or placebo at 14-20 weeks of gestation until delivery. Participants were followed with two pre-delivery visits at gestational week 27-30 and 36-37 and with one post-delivery visit 2-3 days after birth. All visits included blood and fecal sampling. An oral glucose tolerance test was performed at inclusion and gestational week 27-30.

Results: Forty-nine participants completed the study. Thirty-eight participants took more than $80 \%$ of the Vivomixx ${ }^{\circledR}$ capsules $(n=21)$, placebo $(n=17)$. There was no significant difference in HbA1c levels and the occurrence of GDM between groups. There was no significant difference in GWG and infant birth weight between groups in intention to treat analysis. There was, however, a lower mean GWG (11.9 vs $13.0 \mathrm{~kg})$ and lower mean infant birthweight (3554 vs $3658 \mathrm{~g}$ ) in the probiotic group in the per protocol analysis, due to sample size this difference did not reach statistical significance. Fecal microbiota analyses showed an overall increase in a-diversity over time in the Vivomixx ${ }^{\circledR}$ group only $(p=0.016)$.

Conclusions: Administration of probiotics during pregnancy is feasible in obese women. Multi-strain probiotic can modulate the gut microbiota in obese women during pregnancy. A larger study population is needed to uncover whether the results regarding lower GWG and infant birth weight after probiotic supplementation are significant.

Trial registration: ClincalTrials.gov Identifier: NCT02508844, registered on May 11, 2015.

\section{Background}

The increasing prevalence of obesity among women of child-bearing age and during pregnancy has turned into a global public health issue. In 2017, the prevalence of obesity (body mass index (BMI) $\geq 30$ $\mathrm{kg} / \mathrm{m}^{2}$ ) in Denmark was $15 \%$ in women aged $25-34$ years. Obesity in pregnant women increased by $16.4 \%$ between 2004 and 2012 [1]. Likewise, the prevalence of gestational diabetes mellitus (GDM) is increasing in Denmark and internationally $[2,3]$.

Accumulating evidence suggests that high pre-pregnancy BMI is associated with increased risk of developing maternal pregnancy-related complications such as hypertensive disorders, preeclampsia, gestational diabetes mellitus (GDM) and other adverse pregnancy outcomes [4] as well as increased risk of macrosomia, hypoglycemia, and excessive adiposity in the neonate [5,6]. Maternal gestational weight gain (GWG) is associated to the BMI of the offspring and to the risk of obesity in adulthood [7]. A GWG of 
maximum 5-9 kg for obese women is, therefore, recommended in Denmark according to the Institute of Medicine (IOM) guidelines [8].

Data suggest that the composition of the gut microbiota influences body weight, energy homeostasis, insulin resistance, and obesity-associated inflammation, all of which may play a role in the pathophysiology of obesity $[9,10]$. High bacterial diversity and richness is considered a defining factor of a healthy microbiota [11], and a decline in this diversity has been linked to obesity [12].

Probiotics are live microorganisms that, when consumed in adequate amounts, may provide health benefits to the host [13]. Probiotics consist of one or more bacterial species, often including bifidobacteria and lactobacilli, which, during the period of administration, can modify gut microbiota [14]. Probiotics are of particular interest because they are hypothesized to affect body weight, food intake, appetite, and the composition and metabolic functions of the gastrointestinal microbiota $[15,16]$.

Most lifestyle and dietary modification interventions used until now to prevent GDM and excessive GWG during pregnancy have resulted in limited effects $[17,18]$. A systematic review from 2013 reported beneficial effects of probiotics during pregnancy on maternal outcomes including reduced frequency of GDM, improved glycemic control, and a reduced risk of preeclampsia [19]. Most studies, however, have only included normal-weight women.

The number of studies investigating the effect of probiotic supplementation in obese pregnant women on the prevention of GDM and excessive GWG are limited. A randomized controlled trial (RCT) showed no effect on either GWG, maternal fasting glucose, infant birthweight, or other metabolic variables after four weeks of probiotic intervention [20]. A recently published RCT including overweight and obese pregnant women showed a significant effect of probiotics in reducing excessive weight gain versus placebo when administrated from the second trimester until delivery [21]. Fasting glucose measurements were, however, significantly higher in the women receiving probiotics. GDM also occurred more frequent in the probiotic group, but not significantly. This study has no systematic records of gut microbiota monitoring during probiotic intervention in either the obese mothers or their infants.

We hypothesized that probiotic intervention can modulate gut microbiota in obese pregnant women and thereby limit GWG and reduce the risk of adverse maternal and neonatal outcomes. The primary aim of this study was to investigate the feasibility of probiotic intervention in 50 obese pregnant women from the beginning of the second trimester until delivery. Secondary aims were to investigate probiotic effects on maternal and infant perinatal health outcomes, namely GWG, maternal glucose homeostasis (GDM and $\mathrm{HbA} 1 \mathrm{c}$ ), and infant birthweight as well as examine the impact of probiotic intervention on maternal gut microbiota diversity.

\section{Results}

\section{Study population and feasibility}


Fifty of 324 eligible women were included and randomly assigned to Vivomixx ${ }^{\circledR}$ or placebo administration (124 declined participation, 29 were participating in other research projects, and 121 met other exclusion criteria). Of the 50 nulliparous included women, one dropped out during pregnancy and one after delivery (Figure 1). In total, 49 participants (25 Vivomixx ${ }^{\circledR}$ and 24 placebo) were included in ITT analyses and 37 (20 Vivomixx ${ }^{\circledR}$ and 17 placebo) were included in PP analyses. No significant differences between Vivomixx ${ }^{\circledR}$ and placebo participants were found regarding baseline characteristics (Table 1).

Table 1. Baseline characteristics for Vivomixx ® and placebo participants

\begin{tabular}{|l|l|l|l|}
\hline & Vivomixx (n=25) & Placebo (n=25) & $p$-value \\
\hline Pre-pregnancy BMI kg/m², mean (SD) & $31.7(1.8)$ & $32.1(1.3)$ & 0.32 \\
\hline Age in years, mean (SD) & $30.7(4.5)$ & $30.7(4.7)$ & 0.99 \\
\hline Gestational age at baseline in weeks (mean (SD) & $15.5(1.5)$ & $15.1(1.4)$ & 0.36 \\
\hline High risk disposition to GDM*, $n$ (\%) & $7(28)$ & $11(44)$ & 0.38 \\
\hline No. of women reporting smoking during pregnancy & 0 & 1 & NA \\
\hline No. of women reporting smoking at conception & 4 & 2 & NA \\
\hline
\end{tabular}

$\mathrm{BMI}=$ body mass index, GDM = gestational diabetes mellitus, $\mathrm{SD}=$ standard deviation. * family history of diabetes, polycystic ovary syndrome or glycosuria.

Both the probiotic and placebo capsules were widely accepted by the participants. Thirtyeight participants had a capsule intake $>80 \%$ (21 Vivomixx ${ }^{\circledR}$ and 17 placebo). Two participants from the placebo group reported that capsules were difficult to swallow and stopped taking them. Both continued in the study. No other side effects were reported. 
Infant and pregnancy outcomes

GWG at gestational week 36-37 was comparable in the two treatment groups, $(p=0.82)$ (Table 2, Figure 2).

Table 2. Total GWG, intervention period weight gain, and birth weight of infants born to term by women in the Vivomixx@ and placebo group

\begin{tabular}{|c|c|c|c|c|c|c|}
\hline & \multicolumn{3}{|l|}{ ITT } & \multicolumn{3}{|l|}{$\mathrm{PP}$} \\
\hline & Vivomixx (8) & Placebo & $\begin{array}{l}p- \\
\text { value }\end{array}$ & Vivomixx (® & Placebo & $\begin{array}{l}p- \\
\text { value }\end{array}$ \\
\hline $\begin{array}{l}\text { Total GWG, kg (weight in } \\
\text { week } 36-37 \text { minus } \\
\text { prepregnancy weight), mean } \\
\text { (SD) }\end{array}$ & $12.7(5.3)$ & $\begin{array}{l}13.1 \\
(5.8)\end{array}$ & 0.82 & $11.9(4.9)$ & $\begin{array}{l}13.0 \\
(4.2)\end{array}$ & 0.46 \\
\hline $\begin{array}{l}\text { Intervention period weight } \\
\text { gain, kg (weight in week 36- } \\
37 \text { minus weight in week 16- } \\
20 \text { ), mean (SD) }\end{array}$ & $10.2(3.4)$ & $\begin{array}{l}10.0 \\
(4.2)\end{array}$ & 0.87 & $9.9(3.4)$ & $\begin{array}{l}10.8 \\
(3.4)\end{array}$ & 0.44 \\
\hline $\begin{array}{l}\text { Infant birth weight of term- } \\
\text { born infants (q), mean (SD) }\end{array}$ & 3608 (475) & $\begin{array}{l}3640 \\
(454)\end{array}$ & 0.82 & 3554 (487) & $\begin{array}{l}3658 \\
(428)\end{array}$ & 0.52 \\
\hline
\end{tabular}

GWG = gestational weight gain, SD = standard deviation

Four $(16 \%)$ women in the Vivomixx ${ }^{\circledR}$ group and two (8\%) in the placebo group were diagnosed with GDM (IADPSG criteria) in gestational week 27-30 when analyzed in an ITT analysis (Table 3).

Table 3. Glucose values (mmol/L) in fasting state, and 60 and 120 minutes into OGTT at week $14-20$ and at week 27-30 in the Vivomixx $\AA^{\circledR}$ and placebo group. Furthermore, the number of women with a diagnosis of GDM (IADPSG criteria) as well as AUC values for OGTT in the Vivomixx ${ }^{\circledR}$ and placebo group are given. 


\begin{tabular}{|l|l|l|l|l|l|l|}
\hline & $\begin{array}{l}\text { Vivomixx® } \\
(\mathrm{n}=\mathbf{2 5})\end{array}$ & $\begin{array}{l}\text { Placebo } \\
(\mathrm{n}=\mathbf{2 5})\end{array}$ & & $\begin{array}{l}\text { Vivomixx® } \\
(\mathrm{n}=\mathbf{2 5})\end{array}$ & $\begin{array}{l}\text { Placebo } \\
(\mathrm{n}=\mathbf{2 4})\end{array}$ & \\
\hline $\begin{array}{l}\text { OGTT glucose values } \\
\text { mmol/L, } \\
\text { median (IQR) }\end{array}$ & Week (14-20) & Week (14-20) & $\begin{array}{l}\boldsymbol{p} \text { - } \\
\text { value }\end{array}$ & Week 27-30 & Week 27-30 & $\begin{array}{l}\boldsymbol{p} \text { - } \\
\text { value }\end{array}$ \\
\hline 0 & $4.3(4.1: 4.6)$ & $4.4(4.1: 4.6)$ & 0.54 & $4.3(3.9: 4.9)$ & $4.3(4.0: 4.5)$ & 0.85 \\
\hline 60 & $6.9(6.2: 8.0)$ & $6.8(6.3: 8.4)$ & 0.63 & $7.3(6.5: 8.4)$ & $7.3(6.8: 8.7)$ & 0.32 \\
\hline 120 & $6.2(5.4: 6.8)$ & $5.9(5.4: 6.9)$ & 0.41 & $6.3(5.9: 7.1)$ & $6.0(5.3: 6.9)$ & 0.41 \\
\hline GDM diagnosis, $\mathrm{n}(\%)$ & $2(8 \%)$ & $2(8 \%)$ & 1.00 & $4(16 \%)$ & $2(8 \%)$ & 0.67 \\
\hline $\begin{array}{l}\text { OGTT AUC values, } \\
\text { mean (IQR) }\end{array}$ & $\begin{array}{l}732.4 \\
(662.2: 810.8)\end{array}$ & $\begin{array}{l}723.0 \\
(661.5: 820.3)\end{array}$ & 0.83 & $\begin{array}{l}752.7 \\
(674.3: 834.5)\end{array}$ & $\begin{array}{l}743.24 \\
(690.5: 855.4)\end{array}$ & 0.59 \\
\hline
\end{tabular}

$\mathrm{AUC}=$ area under the curve, $\mathrm{GDM}=$ gestational diabetes mellitus, $\mathrm{OGTT}=$ oral glucose tolerance test, $\mathrm{SD}=$ standard deviation. All values are derived from an ITT analysis.

In a PP analysis, the corresponding numbers were four (19\%) and one (5.9\%). The difference in OGTT values when examining the change in area under the curve (AUC) at baseline and gestational week 27-30 was not significantly different in the two groups; Vivomixx ${ }^{\circledR}: 39.19$ (-100.00:127.03) vs. placebo 35.14 (-110.81:186.49), $(p=0.693)$ (Table 3). HbA1c measurements during the intervention period were not significantly different between the two treatment groups, ( $p=0.90$ ) (data not shown). A GWG within the recommended interval of 5-9 kg was achieved in only 6 out of 46 women (13\%). GWG exceeded the IOM guidelines in 38 of 46 women (83\%) (Table 4).

Table 4. Other pregnancy outcomes in the Vivomixx® and placebo group 


\begin{tabular}{|c|c|c|}
\hline & Vivomixx@ $(\mathrm{n}=25)$ & Placebo $(n=24)$ \\
\hline GWG $<5 \mathrm{~kg}, \mathrm{n}$ & 2 & 1 \\
\hline GWG 5-9 kg, n & 2 & 4 \\
\hline $\mathrm{GWG}>9 \mathrm{~kg}, \mathrm{n}$ & 19 & 19 \\
\hline Hypertension, $\mathrm{n}$ & 6 & 5 \\
\hline Preeclampsia, $\mathrm{n}$ & 3 & 3 \\
\hline Induction of labor, $\mathrm{n}$ & 14 & 12 \\
\hline Cesarean section, $\mathrm{n}$ & 11 & 5 \\
\hline Gestational age at birth, days (weeks) & $274(39.2)$ & $280(39.9)$ \\
\hline \multicolumn{3}{|l|}{ Preterm delivery, $\mathrm{n}$} \\
\hline Gestational age 28-34, weeks & 1 & 0 \\
\hline Gestational age 34-37, weeks & 3 & 0 \\
\hline Birth weight (g) all infants, mean (SD) & $3414(676)$ & 3640 (454) \\
\hline Birth weight (g) PP infants, mean (SD) & $3320(703)$ & 3658 (428) \\
\hline$>4 \mathrm{~kg}$ at term, $\mathrm{n}$ & 4 & 7 \\
\hline Small for gestational age (SGA), $n$ & 1 & 0 \\
\hline Large for gestational age (LGA), $\mathrm{n}$ & 1 & 4 \\
\hline Male gender, $\mathrm{n}$ & 13 & 13 \\
\hline Antibiotics during pregnancy, $\mathrm{n}$ & 6 & 5 \\
\hline Antibiotics at birth*, $\mathrm{n}$ & 7 & 12 \\
\hline
\end{tabular}

GWG = gestational weight gain; IOM= Institute of Medicine; PP= per-protocol; SD= standard deviation. * antibiotic treatment at birth was given in cases of premature rupture of the membranes $>18$ hours in accordance with Danish guidelines.

The birth weight of term-born infants was comparable in the two treatment groups, $(p=0.82)$ in the ITT analysis. In the PP analysis mean (SD) birth weight of term-born infants were 3554 (487) vs 3658 (428) in the Vivomixx and placebo groups, $(\mathrm{p}=0.52)$. (Table 2). Four infants were born prematurely, all from mothers in the probiotic group (Table 4). Other pregnancy outcomes are shown in Table 4.

\section{Microbiota results}

$\alpha$-diversity analysis of fecal specimens, showed an increasing diversity in the Vivomixx ${ }^{\circledR}$ group, with a significant difference between the baseline and after birth samples obtained, ( $p=0.016$ ) (Figure 4). No statistically significant differences were observed between any sample times in the placebo group. Bifidobacteria, lactobacilli, and S. salivarius were only found in a fraction of the samples from Vivomixx ${ }^{\circledR}$ treated women, but their relative abundance increased during the intervention period. In samples from Vivomixx ${ }^{\circledR}$ treated women the relative abundance of 
Bifidobacterium was significantly lower in the baseline sample obtained compared to the gestational week 27-30 ( $p=0.021)$, gestational week 36-37 $(p=0.015)$ and after birth $(p=0.020)$ samples. Relative abundance of Lactobacillus was lower in baseline samples compared to gestational week 36-37 and after birth samples $(p=0.03$ and $p=0.02)$, and the relative abundance of $S$. salivarius was found to be significantly higher in the gestational week 36-37 sample compared to baseline and gestational week 2730 samples ( $p=0.0045$ and $p=0.015$ ). Although Bifidobacterium does nominally increase over time in the placebo group there was no significant difference between sample times in the placebo group for either Bifidobacterium, Lactobacillus or S. salivarius.

Samples grouped by cohort and sample times are difficult to distinguish from a simple PCoA plot, however, an analysis of similarities did reveal that Bray-Curtis dissimilarity between baseline samples and samples from gestational week 27-30, gestational week 36-37, and after birth were greater in the Vivomixx ${ }^{\circledR}$ group than in the placebo group $\left(p=3.72 \mathrm{e}^{-6}, p=1.26 \mathrm{e}^{-4}\right.$ and $p=0.0014$ respectively) (Figure 5).

\section{Discussion}

In this randomized double-blinded placebo-controlled study we examined the use of the probiotic supplement Vivomixx ${ }^{\circledR}$ in 50 obese pregnant women. To the best of our knowledge, this study is the first to evaluate the effect of daily multispecies probiotic supplementation on pregnancy outcomes and gut microbiota in pregnant obese women with a long intervention period from gestational week 14-20 until delivery. Forty-nine participants completed the study until delivery, indicating that the study design and intervention is appropriate for further testing. We have shown that Vivomixx® is safe and well tolerated in pregnant women. Dropouts were not due to the capsule intervention. Only two participants in the placebo group stopped taking the capsules due to discomfort when swallowing them. This problem may be solved by using the powdered version of the product, which is also available on the market.

The dietary supplement in this study, Vivomixx®, was chosen for the beneficial effects reported in earlier studies. Vivomixx ${ }^{\circledR}$ consists of eight strains of freeze-dried probiotic bacteria (previously named VSL\#3) and has shown promising results in human trials. A study in 60 overweight $(\mathrm{BMI}>25)$ but otherwise healthy adults showed improved insulin sensitivity $(p<0.01)$, decreased CRP, $(p<0.05)$, and a favorable effect on the gut microbiota after 6 weeks treatment [22]. A randomized study in 48 obese children with nonalcoholic steatohepatitis reported a significantly reduced BMI and an increased GLP-1 secretion after 4 months of treatment compared to placebo $(p<0.001)$ [23]. A small interventional study in pregnant women reported that VSL\#3 administrated during the last trimester of pregnancy was associated with modulation of the vaginal microbiota and cytokine secretion [24]. 
Our data on gut microbiota showed a slight increase in a-diversity during the intervention period in the Vivomixx ${ }^{\circledR}$ group. This was not seen when in the placebo group. Likewise, an increased abundance of lactobacilli, bifidobacterial and Streptococcus salivarius was found in samples from the Vivomixx ${ }^{\circledR}$ group during the intervention period, indicating successful administration. The same was not seen in samples from the placebo group. Equally, principal coordinate analysis indicated that Vivomixx® did result in differences compared to the placebo group.

A PP analysis did show a lower GWG within the intervention period in the probiotic group compared to the placebo group, this difference did, however, not reach statistical significance presumably due to sample size. Callaway et al. did likewise, and in support of our data, show that probiotic intervention had a positive effect on excessive weight gain during pregnancy, where excessive weight gain was seen in $32.5 \%(55 / 169)$ of probiotic treated women versus $46.0 \%(81 / 176)$ of placebo treated women, $(p=0.01)$ [21]. An effect of probiotics on body weight has also been described in non-pregnant overweight and obese women $[25,26]$. In a recent RCT, 6 months of treatment with L. rhamnosus CGMCC1.3724 (LPR) resulted in significant weight loss and reductions in fat mass and circulating leptin concentrations in obese women [25]. In a RCT in 87 adults with a BMI of 24-31, 12 weeks' intake of probiotic milk ( $L$. gasseri SBT2055 (LG2055)) showed significant effect on weight loss $(p<0.001)$, with significant reductions in abdominal, visceral, and subcutaneous fat depots compared to placebo treatment $(p<0.01)$ [26].

Hypotheses about the effects of probiotics in preventing GDM are several, including modulation of glucose tolerance through balancing gut microbiota, normalizing increased intestinal permeability, and lowering systemic and local low-grade inflammation [27]. We found no effect of Vivomixx® on the glucose tolerance of obese women during pregnancy.

The first RCT assessing the efficacy of a probiotic and dietary intervention in reducing the risk of GDM in normal-weight and overweight pregnant women, conducted by Louto et al.,, showed a significantly reduced rate of GDM (13\%) in women receiving both dietary counselling and probiotics (L. rhamnosus GG (ATCC 53103) and B. lactis (BB-12)), ( $p=0.003)$ [28]. Dietary counselling alone had no effect on the rate of GDM (36\%) compared to no intervention (34\%). Other studies have shown inconclusive results. Lindsay et al. conducted a RCT probiotic intervention in 138 obese pregnant women with conflicting results - no difference in the incidence of GDM was found between the probiotic (L. salivarius UCC118) and placebo group [20]. They reported no effect on either the metabolic profile or pregnancy outcomes of their participants. Treatment was, however, only given for 4 weeks [20]. In the study by Callaway et al. reporting a reduction in GWG after probiotic treatment, no probiotic effect was shown on GDM, which occurred in $18.4 \%$ (38/207) of probiotic treated women versus $12.3 \%(25 / 204)$ of placebo treated women, $(p=0.10)$ [21]. Other probiotic interventions including pregnant women diagnosed with GDM are also inconclusive $[29,30]$. Two RCTs including healthy pregnant women have shown that probiotic intervention could maintain serum insulin levels [31] and had significant beneficial effects on markers of insulin metabolism [32]. 
Comparisons between studies is challenging. All the aforementioned studies were conducted with varying probiotic products containing different species and strains. The use of single-versus multi-strain probiotic products can also be discussed. Multi-strain probiotics have been suggested to have improved functionality over single-strain cultures. A newly published study by Forssten et al. reported that probiotics do not have an antagonistic effect on each other's survival when used in a multi-strain product compared to a single-strain product in a simulated colonic environment [33]. In addition, the duration and time of the probiotic intervention during pregnancy varies widely among the studies. Presumably, a longer intervention period would increase the beneficial effects on gut microbiota and metabolism. Furthermore, the effectiveness of probiotic supplements might differ based on the background microbiota compositions of the participants. In addition, studies on the gut microbiota in pregnant women have shown that pregnancy in itself influences the gut microbiota composition, which changes from the first to the third trimester in parallel with weight gain [34,35]. Finally, the reviewed studies were conducted in widely different countries, all with different disease prevalence of e.g. GDM.

The strengths of this study lie in its design (double-blind placebo-controlled study) and the high concentration multi-strain probiotic formulation chosen ( 8 strains, 450 billion CFU per day). Furthermore, the same clinical staff took care of all study visits and participants during the entirety of the study. This ensured continuity, minimized drop-out, and eliminated inter-observer variations in the handling of measurements. A limitation of the study is the low number of study participants, where our sample size was, unfortunately, too small to detect any significant differences in clinical outcomes.

\section{Conclusion}

In this randomized double-blinded placebo-controlled study, we found that intervention with this specific probiotic formulation from gestational week 14-20 until delivery is feasible in obese pregnant women.

A lower mean GWG during pregnancy and lower mean infant birth weight was found in the probiotic group in the per protocol analysis. No significant difference was seen in the occurrence of GDM or HbA1c measurements between the two groups.

Multi-strain probiotic can modulate the gut microbiota in obese women during pregnancy. Microbiota profiling showed a slight increase in a-diversity in women in the Vivomixx ${ }^{\circledR}$ group during the intervention period. Also, an increased abundance of lactobacilli, bifidobacterial and Streptococcus salivarius in a fraction of samples from the Vivomixx ${ }^{\circledR}$ group was found, which indicate successful administration. Likewise, -analysis indicated differences between the Vivomixx ${ }^{\circledR}$ and placebo groups, with some changes in the composition during the intervention period in the Vivomixx ${ }^{\circledR}$ group. These findings need to be confirmed in a larger RCT.

A larger study population is needed to uncover whether the results regarding lower GWG and infant birth weight after probiotic supplementation becomes significant. 


\section{Methods}

\section{Participants}

A randomized controlled study was carried out at Copenhagen University Hospital Hvidovre, Denmark, from February 2015 to January 2018 and included 50 obese pregnant women randomized 1:1 to receive capsules containing Vivomixx ${ }^{\circledR}$ (Visbiome ${ }^{\circledR}$ in North America, DeSimone Formulation ${ }^{\circledR}$ in Asia) or placebo from gestational week 14-20 until delivery. The women and their newborns were followed until nine months after delivery. Participants were identified in connection with the initial nuchal translucency ultrasound scan performed in gestational week 12-14. The study protocol is published in detail elsewhere [36].

The inclusion criteria were as follows: nulliparous singleton pregnant women with $\mathrm{BMI} \geq 30$ and $<35$ $\mathrm{kg} / \mathrm{m}^{2}$ older than 18 years of age; normal nuchal translucency ultrasound scan at gestational age 12-14 weeks; able to read and speak Danish; a successful oral glucose tolerance test (OGTT) at gestational week 14-20.

The exclusion criteria were as follows: gestational age older than 20 weeks at inclusion; pre-gestational diabetes or other serious diseases; multiple pregnancy; previous bariatric surgery; intake of probiotics within the last month before inclusion; ingestion of probiotics during the study intervention period other than the study provided probiotics; alcohol or drug abuse.

\section{Study design}

Participants were included at gestational week 14-20 (baseline) and were followed with clinical visits at gestational week 27-30, gestational week 36-37 and with the newborn 18-72 hours after birth. Fasting blood samples and fecal samples were obtained at each visit and an OGTT was performed at inclusion and at gestational week 27-30.

\section{Randomization}

Participants were randomized 1:1 to receive Vivomixx ${ }^{\circledR}$ capsules or placebo capsules and included by consecutive numbers. Randomization was done in blocks of four. Both Vivomixx ${ }^{\circledR}$ capsules and placebo capsules were identical in appearance and packaging. Investigators, participants, and outcome assessors were blinded to the allocation and intervention. The randomization key was revealed to the researchers only when all participants had completed the 9-month follow-up and data analysis was complete.

\section{Study treatment}


Participants received two capsules of the probiotic mixture Vivomixx® or placebo twice daily. Vivomixx® contains the following strains: Streptococcus thermophilus DSM 24731, bifidobacteria (Bifidobacterium breve DSM 24732, Bifidobacterium longum DSM 24736, Bifidobacterium infantis DSM 24737) and lactobacilli (Lactobacillus acidophilus DSM 24735, Lactobacillus plantarum DSM 24730, Lactobacillus paracasei DSM 24733, Lactobacillus delbrueckii subsp. bulgaricus DSM 24734), and is formulated in vegetable capsules containing 112 billion lyophilized bacteria.

\section{Pregnancy outcomes}

GWG was defined as body weight at gestational week 36-37 minus self-reported pre-pregnancy body weight. Participants were weighed using the same scale at every study visit, wearing light clothes and no shoes.

A two-hour three-time-point 75g OGTT was carried out in gestational week 14-20 (baseline) and in gestational week 27-30. Diagnosis of GDM was defined according to the International Association of Diabetes in Pregnancy Study Group (IADPSG) criteria [37]; the diagnosis was established if one or more glucose values were above the following values: 0 -value $\geq 5.1 \mathrm{mmol} / \mathrm{L} ; 60 \mathrm{~min}$-value $\geq 10.0 \mathrm{mmol} / \mathrm{L}$; 120 min-value $\geq 8.5 \mathrm{mmol} / \mathrm{L}$. GDM in Danish routine pregnancy care was diagnosed using the Danish national guidelines (DSOG guidelines). According to these guidelines, GDM is diagnosed if the 2-hour standard OGTT capillary blood glucose is $9 \mathrm{mmol} / \mathrm{L}$ or greater. Participants diagnosed with GDM according to Danish guidelines continued their care in the multidisciplinary diabetic clinic but were not excluded from the study.

Data on pregnancy-related complications and mode of delivery were extracted from hospital files and validated using the DSOG guidelines; preeclampsia was diagnosed if participants had proteinuria (dipstick, greater than 1+ protein) and persistently elevated blood pressure greater than $140 / 90 \mathrm{mmHg}$ on more than one occasion. Gestational hypertension was diagnosed using the same criteria but without proteinuria.

\section{Neonatal outcomes}

Neonatal outcomes included gestational age, birth weight, and birth length and were obtained from hospital records. Infants with a gestational age less than 38 weeks were excluded from analyses involving anthropological measurements. A term-born neonate was considered large for gestational age (LGA) when weighing over $4000 \mathrm{~g}$ and small for gestational age (SGA) when weighing under $2500 \mathrm{~g}$ $[38,39]$.

\section{Microbiota diversity analysis}


Microbiota diversity analysis relied on sequencing of ribosomal small subunit (SSU rRNA) genes. Purified genomic DNA was submitted to PCR using four different primer sets, targeting prokaryotes (one primer pair) and eukaryotes (three primer pairs). For prokaryotes, a modified version of the published universal prokaryotic primers 341F/806R [40] were used, while three different in-house primers (G3F1/G3R1, G4F3/G4R3, and G6F1/G6R1) were used to amplify eukaryotic DNA $[41,42]$. Resulting PCR products were quantified using the Quant-IT ${ }^{\mathrm{TM}}$ dsDNA High Sensitive Assay Kit (Thermo Fisher Scientific) and pooled in equimolar amounts (PAL: Pooled Amplicon Library). Agencourt AMPure XP Beads (Beckman Coulter) were used to remove DNA fragments shorter than $300 \mathrm{bp}$ and those longer than 1,000 bp, and the purified DNA was sequenced on the Illumina MiSeq system in a 2 x 250 bp setup (Illumina Inc., San Diego, CA 29122, USA). A maximum of 64 samples were sequenced in a single sequencing run $[41,42]$.

The sequence output was taxonomically mapped using BION, a newly developed k-mer-based mapping software. A k-mer length of eight was used, with a step size of four. Query sequences originating from prokaryotes were compared with the 340-807 bp region (rRNA gene positions from Escherichia coli) in RDP 11.04 [43], and eukaryotic query sequences were compared against the same region in SILVA version 123 [44] with an in-house-improved taxonomy for eukaryotes.

\section{Statistical methods}

All statistical analyses were performed in R 3.2.3 [45]. Continuous variables are represented as mean values and standard deviations. Non-normally distributed variables are represented as median values and interquartile ranges. Continuous variables were compared using $t$ tests or, for non-normally distributed data, the Wilcoxon sum rank test. Categorical variables were presented as frequencies and compared using the chi-squared test or Fisher's exact test. A $p$-value of $<0.05$ was considered statistically significant. Statistical analysis of microbiota was performed with the phyloseq and vegan packages, using ggplot2 and plotly for data visualization. Comparisons of a-diversity and relative abundance of specific OTUs across sample times and between cohorts were done using Mann-Whitney U tests. Analysis of similarities were performed based on Bray-Curtis dissimilarity between samples.

Both intention-to-treat (ITT) and per-protocol (PP) analyses were performed. ITT analyses included all study participants. PP analyses included all participants with a compliance of more than $80 \%$ capsule intake and excluded all participants diagnosed with GDM according to Danish national guidelines, these were excluded to avoid possible bias related to the additional consultations with health care professionals, including dietician counseling, offered to these participants.

\section{Abbreviations}

BMI: Body mass index; GDM: Gestational diabetes mellitus; GWG: Gestational weight gain; HbA1c: glycated hemoglobin; IADPSG: International Association of Diabetes in Pregnancy Study Group; IOM: Institute of Medicine; ITT: Intention-to-treat; LGA: Large for gestational age; OGTT: Oral glucose tolerance test; PP: Per-protocol; RCT: Randomized controlled trial; SGA: Small for gestational age 


\section{Declarations}

\section{Acknowledgments}

The authors wish to express their gratitude to all the women and their infants who participated in the study and to all midwives and other hospital staff who accommodated the study into their daily work functions. We also wish to thank the clinical microbiology laboratory staff for help with handling the fecal samples.

\section{Funding}

The study was financed by grants from the following private foundations; Jeppe Juhls og hustru Ovita Juhls Mindelegat, Else og Mogens Wedell-Wedellborgs Fond, Aase og Ejnar Danielsens Fond, Knud og Edith Eriksens Mindefond, Toyota-Fonden Denmark, and Next Gen Pharma India Pvt. Ltd. The study was co-financed by the Faculty of Health and Medical Sciences, University of Copenhagen. All funding sources had no role in the study design, data collection, interpretation of analyses, writing of the manuscript, or decision to submit the publication.

\section{Availability of data and materials}

The data are available from the authors at request.

\section{Authors' contributions}

Halkjær SI, Nilas L, Cortes D, Krogfelt KA, Pedersen AE and Petersen AM designed the research project; Halkjær SI and de Knegt VE performed the clinical research; Lo B designed the database; Halkjær SI, de Knegt VE and Lo B entered data; Kallemose T and Johannesen TB performed the statistical analysis; Andersen LO, Mirsepasi-Lauridsen HC, Stensvold CR, Nielsen HV and Johannesen TB performed the sequencing and microbiota analysis; Halkjær SI wrote the paper and co-authors supervised the paper; all authors read, commented and approved the final manuscript.

\section{Ethics approval and consent to participate}

The study was approved by the Danish Data Protection Agency (AHH-2015-001), and permission for human experiments and recruitment of participants was obtained from the Scientific Ethics Committee for Copenhagen Regional Hospitals, Denmark (Permission no.: H-2-2014-076) version 2.1-5 December 2014. The study was performed in accordance with the Revised Declaration of Helsinki. The study was registered in www.clinicaltrials.gov (NCT02508844). All participants provided written informed consent to participate after verbal and written information was given. Participants were informed that they could withdraw from the study at any time. 


\section{Consent for publication}

All authors of the manuscript have read and agreed to its content and are accountable for all aspects of the accuracy and integrity of the manuscript in accordance with ICMJE criteria. The article is original, has not already been published in a journal, and is not currently under consideration by another journal.

\section{Competing interests}

The authors declare that they have no competing interests.

Both the probiotic and placebo capsules as well as half a year's salary to the study have been donated to Copenhagen University Hospital Hvidovre by Next Gen Pharma India Pvt. Ltd., H. No. 331, 1st Floor, Sector 15A, Noida 201301, National Capital Region, India. The funding source had no role in the study design, data collection, interpretation of analysis, writing of the manuscript, or decision to submit the publication.

\section{References}

1 Iversen DS, Kesmodel US, Ovesen PG. Associations between parity and maternal BMI in a populationbased cohort study. Acta Obstet Gynecol Scand 2018;97:694-700. doi:10.1111/aogs.13321

2 Ovesen PG, Fuglsang J, Andersen MB, et al. Temporal Trends in Gestational Diabetes Prevalence, Treatment, and Outcomes at Aarhus University Hospital, Skejby, between 2004 and 2016. J Diabetes Res 2018;2018:5937059. doi:10.1155/2018/5937059

3 Ferrara A. Increasing prevalence of gestational diabetes mellitus: a public health perspective. Diabetes Care 2007;30 Supp/ 2:S141-146. doi:10.2337/dc07-s206

4 Ovesen P, Rasmussen S, Kesmodel U. Effect of prepregnancy maternal overweight and obesity on pregnancy outcome. Obstet Gynecol 2011;118.305-12. doi:10.1097/AOG.0b013e3182245d49

5 Catalano PM, McIntyre HD, Cruickshank JK, et al. The hyperglycemia and adverse pregnancy outcome study: associations of GDM and obesity with pregnancy outcomes. Diabetes Care 2012;35:780-6. doi:10.2337/dc11-1790

6 Ludwig DS, Currie J. The association between pregnancy weight gain and birthweight: a within-family comparison. Lancet Lond Eng/2010;376.984-90. doi:10.1016/S0140-6736(10)60751-9

7 Schack-Nielsen L, Michaelsen KF, Gamborg M, et al. Gestational weight gain in relation to offspring body mass index and obesity from infancy through adulthood. Int J Obes 2009;34:67-74. doi:10.1038/ijo.2009.206

8 Institute of Medicine (US) and National Research Council (US) Committee to Reexamine IOM Pregnancy Weight Guidelines. Weight Gain During Pregnancy: Reexamining the Guidelines. Washington (DC):: 
National Academies Press (US) 2009. http://www.ncbi.nlm.nih.gov/books/NBK32813/ (accessed 25 Jul 2018).

9 Bäckhed F, Ding $\mathrm{H}$, Wang $\mathrm{T}$, et al. The gut microbiota as an environmental factor that regulates fat storage. Proc Natl Acad Sci U S A 2004;101:15718-23. doi:10.1073/pnas.0407076101

10 Kobyliak N, Conte C, Cammarota G, et al. Probiotics in prevention and treatment of obesity: a critical view. Nutr Metab 2016;13:14. doi:10.1186/s12986-016-0067-0

11 Bäckhed F, Fraser CM, Ringel Y, et al. Defining a healthy human gut microbiome: current concepts, future directions, and clinical applications. Cell Host Microbe 2012;12:611-22.

doi:10.1016/j.chom.2012.10.012

12 Le Chatelier E, Nielsen T, Qin J, et al. Richness of human gut microbiome correlates with metabolic markers. Nature 2013;500:541-6. doi:10.1038/nature12506

13 Hill C, Guarner F, Reid G, et al. Expert consensus document. The International Scientific Association for Probiotics and Prebiotics consensus statement on the scope and appropriate use of the term probiotic. Nat Rev Gastroenterol Hepatol 2014;11:506-14. doi:10.1038/nrgastro.2014.66

14 Delzenne NM, Neyrinck AM, Bäckhed F, et al. Targeting gut microbiota in obesity: effects of prebiotics and probiotics. Nat Rev Endocrino/ 2011;7:639-46. doi:10.1038/nrendo.2011.126

15 Sanchez M, Panahi S, Tremblay A. Childhood obesity: a role for gut microbiota? Int J Environ Res Public Health 2014;12:162-75. doi:10.3390/ijerph120100162

16 Kang Y, Cai Y. The development of probiotics therapy to obesity: a therapy that has gained considerable momentum. Horm Athens Greece 2018;17:141-51. doi:10.1007/s42000-018-0003-y

17 Morisset A-S, St-Yves A, Veillette J, et al. Prevention of gestational diabetes mellitus: a review of studies on weight management. Diabetes Metab Res Rev 2010;26:17-25. doi:10.1002/dmrr.1053

18 Egan AM, Simmons D. Lessons learned from lifestyle prevention trials in gestational diabetes mellitus. Diabet Med J Br Diabet Assoc 2019;36:142-50. doi:10.1111/dme.13772

19 Lindsay KL, Walsh CA, Brennan L, et al. Probiotics in pregnancy and maternal outcomes: a systematic review. J Matern-Fetal Neonatal Med Off J Eur Assoc Perinat Med Fed Asia Ocean Perinat Soc Int Soc Perinat Obstet 2013;26:772-8. doi:10.3109/14767058.2012.755166

20 Lindsay KL, Kennelly M, Culliton M, et al. Probiotics in obese pregnancy do not reduce maternal fasting glucose: a double-blind, placebo-controlled, randomized trial (Probiotics in Pregnancy Study). Am J Clin Nutr 2014;99:1432-9. doi:10.3945/ajcn.113.079723 
21 Callaway LK, Mclntyre HD, Barrett HL, et al. Probiotics for the Prevention of Gestational Diabetes Mellitus in Overweight and Obese Women: Findings From the SPRING Double-Blind Randomized Controlled Trial. Diabetes Care 2019;42:364-71. doi:10.2337/dc18-2248

22 Rajkumar H, Mahmood N, Kumar M, et al. Effect of probiotic (VSL\#3) and omega-3 on lipid profile, insulin sensitivity, inflammatory markers, and gut colonization in overweight adults: a randomized, controlled trial. Mediators Inflamm 2014;2014.348959. doi:10.1155/2014/348959

23 Alisi A, Bedogni G, Baviera G, et al. Randomised clinical trial: The beneficial effects of VSL\#3 in obese children with non-alcoholic steatohepatitis. Aliment Pharmacol Ther 2014;39:1276-85. doi:10.1111/apt.12758

24 Vitali B, Cruciani F, Baldassarre ME, et al. Dietary supplementation with probiotics during late pregnancy: outcome on vaginal microbiota and cytokine secretion. BMC Microbio/ 2012;12.236. doi:10.1186/1471-2180-12-236

25 Sanchez M, Darimont C, Drapeau V, et al. Effect of Lactobacillus rhamnosus CGMCC1.3724 supplementation on weight loss and maintenance in obese men and women. Br J Nutr 2014;111:150719. doi:10.1017/S0007114513003875

26 Kadooka Y, Sato M, Imaizumi K, et al. Regulation of abdominal adiposity by probiotics (Lactobacillus gasseri SBT2055) in adults with obese tendencies in a randomized controlled trial. Eur J Clin Nutr 2010;64:636-43. doi:10.1038/ejcn.2010.19

27 Isolauri E, Rautava S, Collado MC, et al. Role of probiotics in reducing the risk of gestational diabetes. Diabetes Obes Metab 2015;17:713-9. doi:10.1111/dom.12475

28 Luoto R, Laitinen K, Nermes M, et al. Impact of maternal probiotic-supplemented dietary counselling on pregnancy outcome and prenatal and postnatal growth: a double-blind, placebo-controlled study. $\mathrm{Br} J$ Nutr 2010;103:1792-9. doi:10.1017/S0007114509993898

29 Lindsay KL, Brennan L, Kennelly MA, et al. Impact of probiotics in women with gestational diabetes mellitus on metabolic health: a randomized controlled trial. Am J Obstet Gynecol 2015;212:496.e1-11. doi:10.1016/j.ajog.2015.02.008

30 Kijmanawat A, Panburana P, Reutrakul S, et al. Effects of probiotic supplements on insulin resistance in gestational diabetes mellitus: A double-blind randomized controlled trial. J Diabetes Investig 2019;10:163-70. doi:10.1111/jdi.12863

31 Asemi Z, Samimi M, Tabassi Z, et al. Effect of daily consumption of probiotic yoghurt on insulin resistance in pregnant women: a randomized controlled trial. Eur J Clin Nutr 2013;67:71-4. doi:10.1038/ejcn.2012.189 
32 Jamilian M, Bahmani F, Vahedpoor Z, et al. Effects of Probiotic Supplementation on Metabolic Status in Pregnant Women: a Randomized, Double-blind, Placebo-Controlled Trial. Arch Iran Med 2016;19:687682. doi:0161910/AIM.004

33 Forssten SD, Ouwehand AC. Simulating colonic survival of probiotics in single-strain products compared to multi-strain products. Microb Ecol Health Dis 2017;28:1378061.

doi:10.1080/16512235.2017.1378061

34 Koren O, Goodrich JK, Cullender TC, et al. Host remodeling of the gut microbiome and metabolic changes during pregnancy. Cell 2012;150:470-80. doi:10.1016/j.cell.2012.07.008

35 Collado MC, Isolauri E, Laitinen $\mathrm{K}$, et al. Effect of mother's weight on infant's microbiota acquisition, composition, and activity during early infancy: a prospective follow-up study initiated in early pregnancy. Am J Clin Nutr 2010;92:1023-30. doi:10.3945/ajcn.2010.29877

36 Halkjaer SI, Nilas L, Carlsen EM, et al. Effects of probiotics (Vivomixx®) in obese pregnant women and their newborn: study protocol for a randomized controlled trial. Trials 2016;17:491. doi:10.1186/s13063016-1617-5

37 International Association of Diabetes and Pregnancy Study Groups Consensus Panel, Metzger BE, Gabbe SG, et al. International association of diabetes and pregnancy study groups recommendations on the diagnosis and classification of hyperglycemia in pregnancy. Diabetes Care 2010;33:676-82. doi:10.2337/dc09-1848

38 Lawrence EJ. Part 1: a matter of size: evaluating the growth-restricted neonate. Adv Neonatal Care Off J Natl Assoc Neonatal Nurses 2006;6:313-22. doi:10.1016/j.adnc.2006.08.006

39 Lawrence EJ. A matter of size: Part 2. Evaluating the large-for-gestational-age neonate. Adv Neonatal Care Off J Natl Assoc Neonatal Nurses 2007;7:187-97; quiz 198-9. doi:10.1097/01.ANC.0000286335.06047.28

40 Yu Y, Lee C, Kim J, et al. Group-specific primer and probe sets to detect methanogenic communities using quantitative real-time polymerase chain reaction. Biotechnol Bioeng 2005;89:670-9. doi:10.1002/bit.20347

41 Ring HC, Thorsen J, Saunte DM, et al. The Follicular Skin Microbiome in Patients With Hidradenitis Suppurativa and Healthy Controls. JAMA Dermato/ 2017;153:897-905.

doi:10.1001/jamadermatol.2017.0904

42 Krogsgaard LR, Andersen LO 'Brien, Johannesen TB, et al. Characteristics of the bacterial microbiome in association with common intestinal parasites in irritable bowel syndrome. Clin Trans/ Gastroenterol 2018;9:161. doi:10.1038/s41424-018-0027-2 
43 Cole JR, Wang Q, Fish JA, et al. Ribosomal Database Project: data and tools for high throughput rRNA analysis. Nucleic Acids Res 2014;42:D633-42. doi:10.1093/nar/gkt1244

44 Yilmaz P, Parfrey LW, Yarza P, et al. The SILVA and "All-species Living Tree Project (LTP)" taxonomic frameworks. Nucleic Acids Res 2014;42:D643-8. doi:10.1093/nar/gkt1209

45 R: The R Project for Statistical Computing. https://www.r-project.org/ (accessed 25 Oct 2018).

Figures 


\section{$\Rightarrow$ CONSORT}

TRANSPARENT REPORTING Of TRIALS

\section{CONSORT 2010 Flow Diagram}

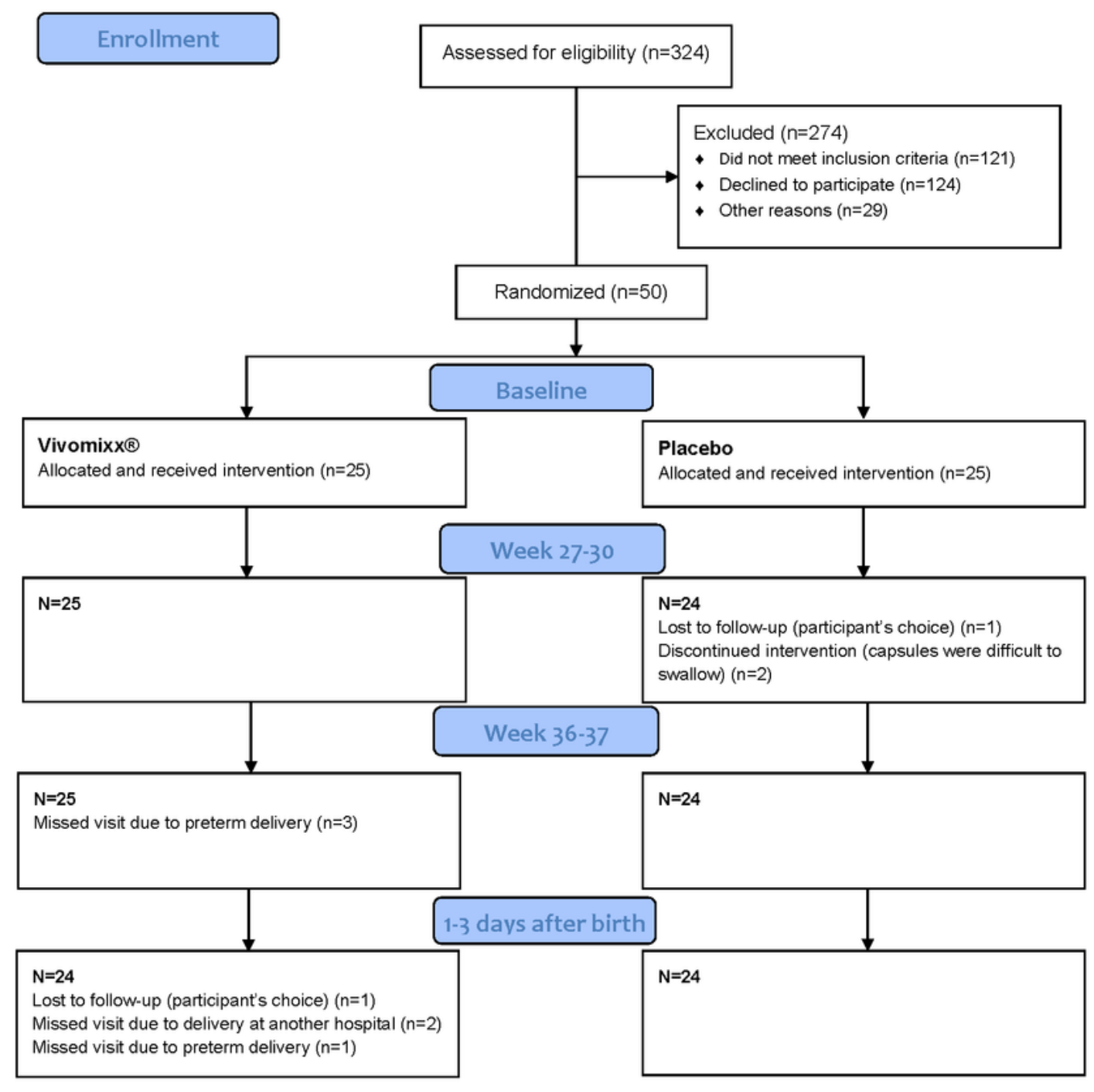

\section{Figure 1}

CONSORT Flow Diagram. Flow diagram of inclusion and study visits for participants randomized to Vivomixx ${ }^{\circledR}$ and placebo group. Forty-nine participants completed the study until delivery. Participants with missed visits due to preterm delivery or discontinued capsule intervention where not excluded from following study visits 


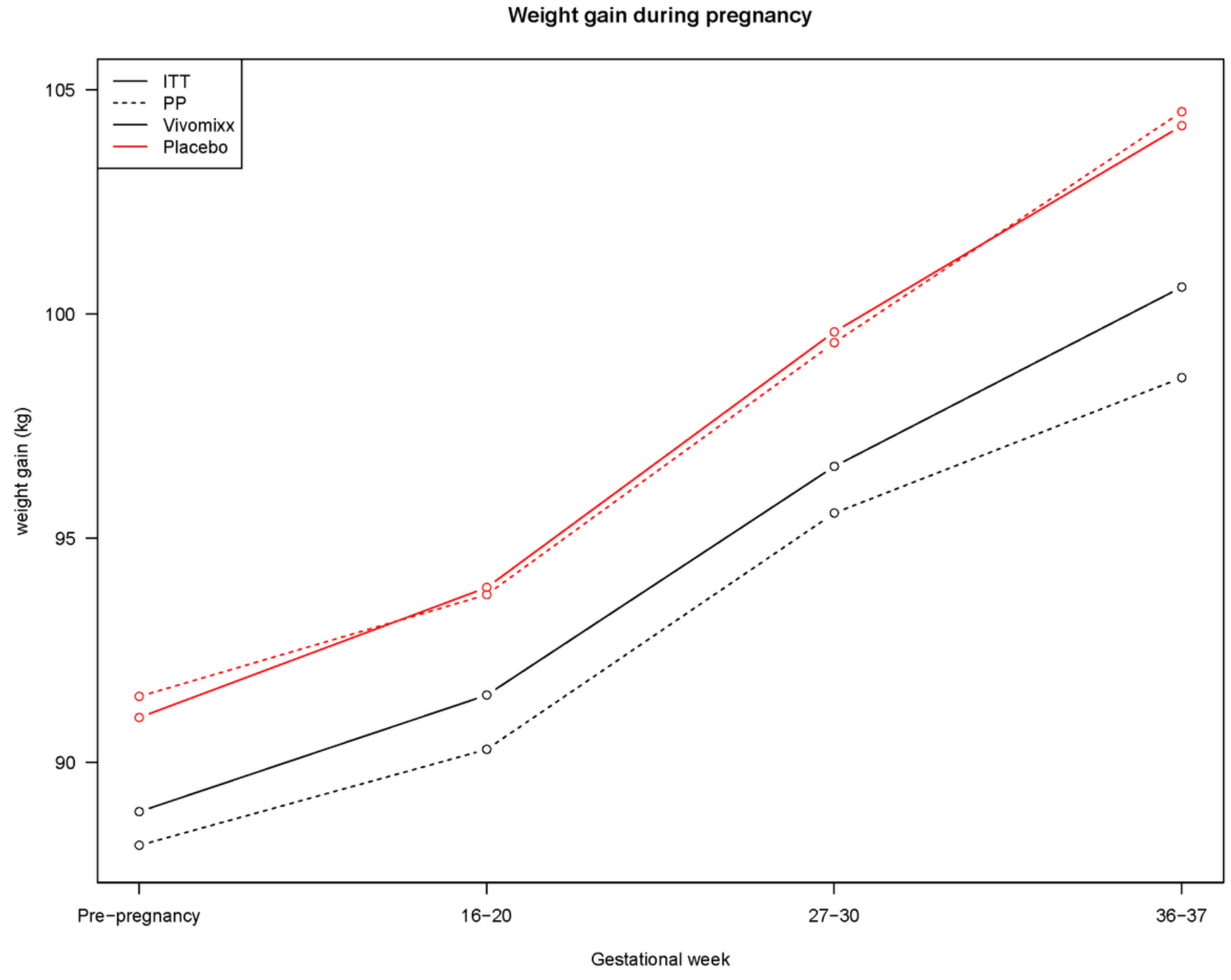

Figure 2

Gestational weight gain (GWG) in Vivomixx® and placebo group. 


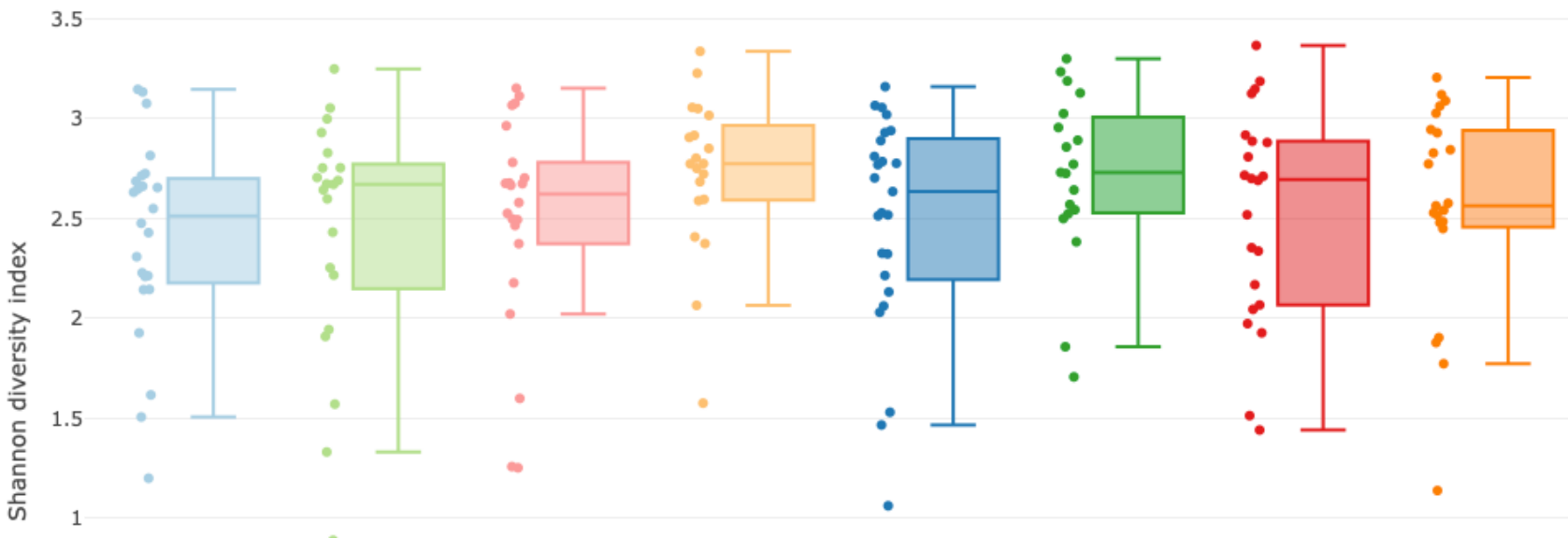

0.5

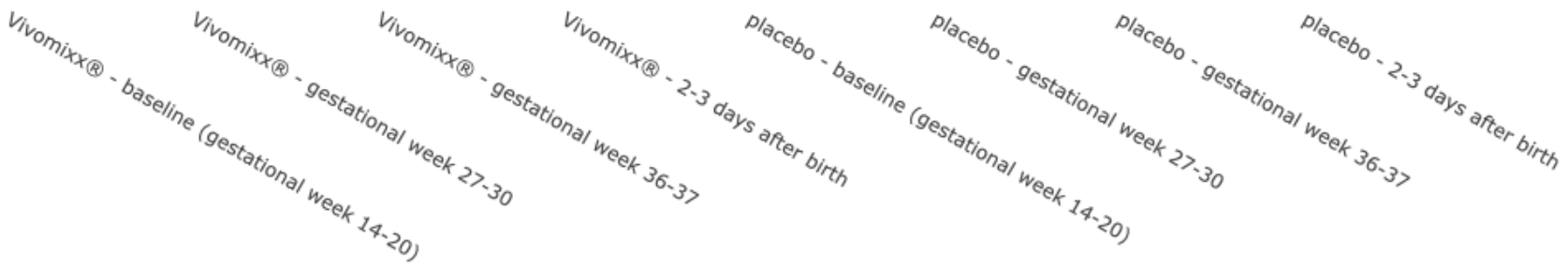

Figure 3

Change in a-diversity over time in the Vivomixx ${ }^{\circledR}$ and placebo group. Blue= baseline sample, gestational week 14-20; green= gestational week 27-30; red= gestational week 36-37; orange $=2-3$ days after birth 

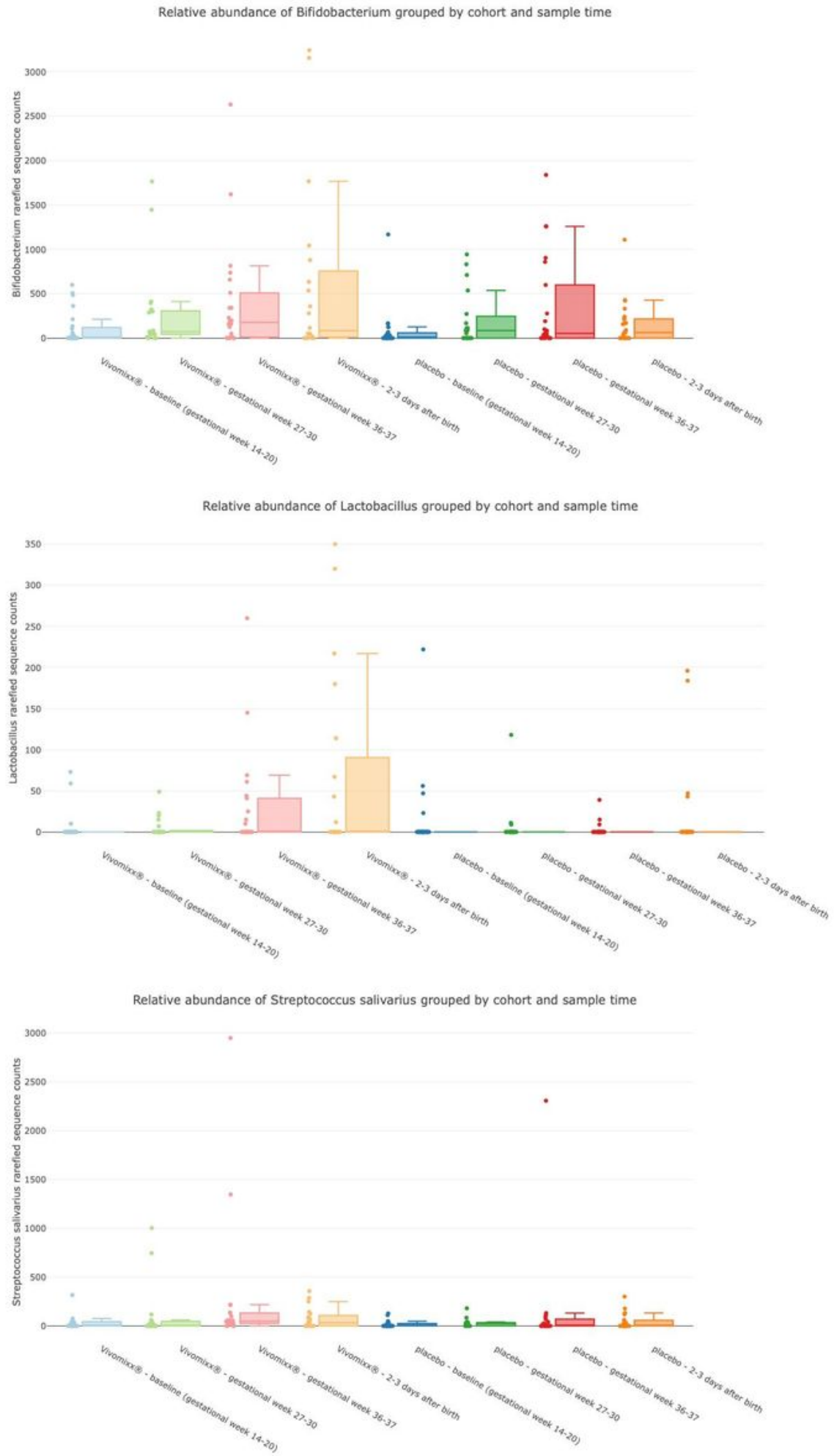

\section{Figure 4}

Relative abundance of Bifidobacterium, Lactobacilli and Streptococcus salivarius (y-axis) in the Vivomixx ${ }^{\circledR}$ and placebo group. X-axis: bluer= Vivomixx ${ }^{\circledR} /$ placebo baseline, gestational week 14-20; green= Vivomixx $\AA /$ placebo gestational week 27-30; red=Vivomixx $® /$ placebo gestational week 36-37; blue= Vivomixx ${ }^{\circledR} /$ placebo 2-3 days after birth 


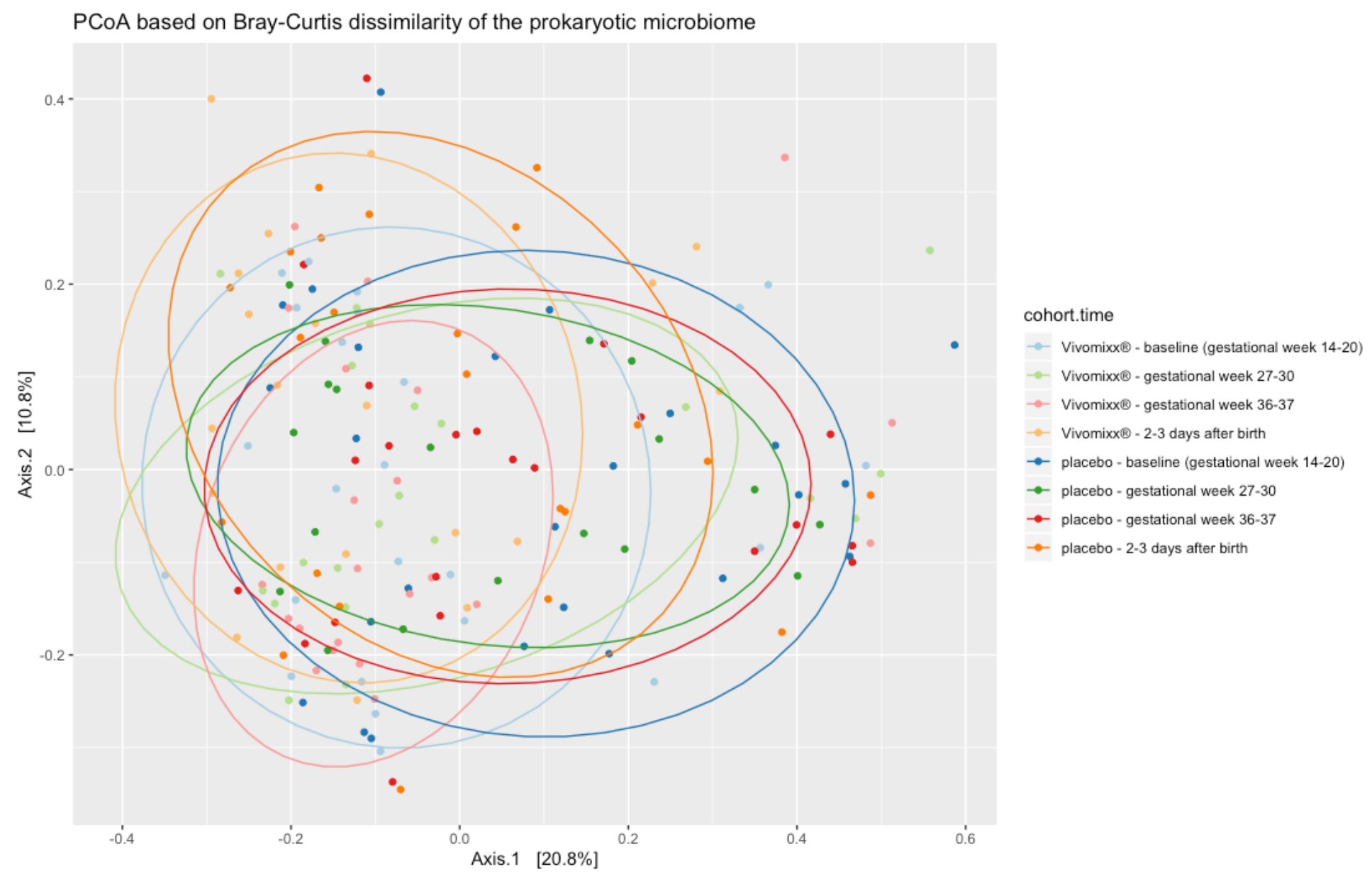

Figure 5

Principal coordinate analysis on the prokaryotic microbiome in the Vivomixx $\circledast$ and placebo group during the study. Blue= baseline, gestational week 14-20; green= gestational week 27-30; red= gestational week $36-37$; orange $=2-3$ days after birth 